\title{
Reflexiones sobre el vínculo pedagógico: Evaluación formativa con algunos participantes de proyecto de formación y divulgación de Capoeira de la corporación $\mathrm{X}$
}

\author{
Reflections on the pedagogical bond: \\ Formative evaluation with some participants of the project \\ of formation and divulgation of Capoeira of the X corporation
}

\section{Diego Ortiz Morales}

Universidad Pontificia Bolicariana, Colombia

Correo: formarcrea@gmail.com

Resumen: Como un paso indispensable para el crecimiento y mejora de los procesos pedagógicos de la Corporación Mangle, se propone realizar un proceso de evaluación formativa con algunos de los participantes de su proyecto de formación en Capoeira. Con tal fin se diseña una herramienta, que, busca identificar las transformaciones auto percibidas por dichos participantes, a partir de entrevistas en profundidad acompañadas por un análisis hermenéutico, dicho análisis se realizó a la luz de un marco de análisis elaborado a partir de la intención pedagógica del proyecto en cuestión, la cual fue identificada a partir de tres entrevistas semiestructuradas con la fundadora de la Corporación y una revisión bibliográfica de la misma. Los participantes no solo manifestaron las transformaciones auto percibidas, sino que, además, comenzaron a hacer evidente los mecanismos que hicieron posible dichas transformaciones, entre ellos el más relevante en sus discursos fue llamado vínculo pedagógico, es decir la red de relaciones que se teje al interior de la comunidad educativa, a saber, profesores, estudiantes, familia y contexto. Este vínculo, tejido gracias a la admiración, la autoridad, la camaradería y el compartir de una pasión común se concibe entonces como pilar del proceso de formación integral del ser humano.

Palabras clave: auto percepción, capoeira, formación integral, intención pedagógica, vínculo pedagógico

\begin{abstract}
As an indispensable step for the growth and improvement of the Mangle Corporation's pedagogical processes, it is proposed to carry out a formative evaluation process with some of the participants of its Capoeira training project. To this end, a tool is designed that seeks to identify the transformations that these participants perceive in themselves, based on in-depth interviews accompanied by a hermeneutic analysis. This analysis was done in light of a framework of analysis developed from the pedagogical intention of the project in question, which was identified from three semi-structured interviews with the Corporation's founder and a bibliographic review of the same. The participants not only manifested the self-perceived transformations, but also began to make evident the mechanisms that made these transformations possible. Among them, the most relevant in their speeches was called the pedagogical bond, that is, the network of relationships that is woven within the educational community, namely, teachers, students, family and context. This bond, woven through admiration, authority, camaraderie, and the sharing of a common passion, is then conceived as a pillar of the process of the integral formation of the human being.
\end{abstract}

Keywords: capoeira, integral formation, self-perception, pedagogic bond, pedagogic intention 


\section{Introducción}

Cuando se propone un verbo para el objetivo de la investigación cuyos resultados se presentan a continuación, se evidenció un dilema: ¿Describir o evaluar? Lo cual, desenmascaró cuestiones éticas y filosóficas subyacentes al acto de investigar. Plantear el verbo obligó a cuestionar sobre las motivaciones subyacentes a la investigación, y sobre algo fundamental: ¿Para quién se investiga?

Es vital reconocer que quienes perciben el mayor beneficio por la realización de una investigación social es la comunidad o las personas con quienes se realiza la investigación. Más aún, esta adquiere valor cuando la concebimos como un proceso de investigación participativa, de construcción conjunta de saberes, en el que las personas se involucran y, por ende, se transforman. Por ello, se decidió encabezar el objetivo con el verbo evaluar, entendiendo a la evaluación como un paso clave dentro del proceso de formación. La evaluación, además de medir el logro o fracaso en el aprendizaje, es un paso fundamental dentro del proceso formativo. Es decir que, dicho proceso sin una etapa de evaluación, en el sentido que aquí se concibe, es un proceso inacabado.

Los resultados que se presentan, entonces, no sólo pretenden describir los hallazgos en un ejercicio académico y conceptual, sino que, además, buscan aportar a la Corporación X, con una evaluación formativa de su proyecto de formación en Capoeira. En relación con la evaluación formativa, Fernández (2017) nos dice que: "El objetivo no se centra en dar una calificación final sino en favorecer, confirmar o rectificar el método y orientar al aprendiz sobre la misma marcha para progresar." (p. 4).

¿Cómo se llega a ser lo que se es?

En el centro de la ciudad de Medellín se extiende un territorio de aproximadamente 42 hectáreas, que, en poco más de 30 años, pasó de ser un potrero a ser el barrio más densamente poblado de Colombia y el segundo de este ranking en Latinoamérica: Moravia (Ramírez, 2019). A partir de 1977 la municipalidad decidió ubicar allí el botadero de basura de la ciudad, copándolo por completo en 1984. Sin embargo, cientos de familias que habían huido de la violencia que azotaba la zona norte y suroccidente del país, encontraron en la basura no sólo su hogar sino su fuente de empleo. Lo que para sus vecinos era basura para ellos se convirtió en un tesoro.

De la mano del sacerdote Vicente Mejía Espinoza, los habitantes del barrio comenzaron a trazar las calles, la escuela, la cancha, la iglesia y el alcantarillado público, resistiéndose al desalojo y a la demolición de las viviendas. Una de las estrategias era hacer una eucaristía cada que llegaba la fuerza pública, quienes interrumpían el operativo por respeto a la celebración. Este espíritu de resistencia creó una conciencia política al interior del barrio y un liderazgo efectivo que los llevo, en 2008, de la mano de la alcaldía, Comfenalco Antioquia y un donante anónimo, a construir el Centro de Desarrollo Cultural de Moravia, obra póstuma del maestro Rogelio Salmona. Esta obra, pronto se convertiría en un referente para la cultura de la ciudad.

Es allí, en el barrio Moravia en dónde nace la corporación X en el 2007. Esta corporación inició como un grupo de amigos y amigas que buscaban un lugar para cultivar su pasión por la Capoeira y que, con el tiempo, pasarían a convertirse en un modelo de gestión cultural, gracias a alianzas nacionales e internacionales ${ }^{1}$. Gracias a estas alianzas se gesta el proyecto de formación y divulgación de Capoeira, una práctica de resistencia y memoria que se encuentra en Moravia. Un proyecto que ha sido el proyecto más grande de la Corporación, pues, ha permitido que tenga presencia en el barrio

${ }^{1}$ Con entidades como Comfenalco Antioquia, Fundación EPM, Fundación patronato de María Auxiliadora, Organización de Estados Iberoamericanos (OEI) y el grupo Oficina da Capoeira Internacional. 
Moravia y otros barrios de Medellín y Antioquia. Habló su nicho, resonando en los habitantes de un barrio que, frente a la fatal amenaza del olvido y el desarraigo opta por la lucha y la colectividad como formas de resistencia.

Al comenzar a vislumbrar transformaciones tras las que se había planteado todo el esquema de investigación comenzaron a emerger mecanismos, formas en que dichas transformaciones se habían dado. La pregunta por la transformación comenzó a relacionarse con la pregunta por el acto formativo y las relaciones que se entablan entre "la profe" y el alumno. La evaluación formativa comienza así a involucrarse con la propuesta pedagógica de la Corporación, revelando la importancia de las decisiones, motivaciones y actos que subyacen al acto de enseñar. Una evaluación multidimensional, interpersonal e intrapersonal, ya que habla de la volición, la cognición, el deseo, la afectividad, los valores, la imaginación y la trascendencia, tejiendo relaciones entre las dimensiones del ser y entre los universos que en el acto pedagógico se encuentran: «la profe», los compañeros, la familia, el barrio y la tradición (ancestralidad). Es por esto por lo que cada uno de los objetivos específicos se entabla como una forma de explorar las diferentes voces que participan en el proceso formativo, pero al mismo tiempo buscan integrarlas.

Los hallazgos que se presentan hablan del "Qué", es decir, las transformaciones percibidas, que en ocasiones son halladas de forma literal en las historias y opiniones que los participantes expresan en torno a sus experiencias, con frases como "Sí, yo creo que tanto la Capoeira como el colegio fueron como los que me quitaron la timidez, porque en Capoeira yo era de los menores, había muchos peques, pero hicimos un grupo de amigos y fue muy chévere y ahí fui soltando" (Emmanuel. Entrevista. 27 de marzo de 2020). Sin embargo, a partir de estas historias se comienza a tejer en el análisis hermenéutico, las formas del acto formativo. Nos comenzamos a sentir cuestionados por el "Cómo". Sin embargo, este "Cómo" debe ser observado a la luz del marco teórico y en ocasiones soportado por observaciones comparativas entre las entrevistas de los diferentes informantes.

Afirmar por ejemplo que Duván admira a Camero es posible, ya que se revela de forma literal en una frase dicha por Duván: "Camero, es que yo lo admiro mucho hermano" (Duván. Entrevista. 4 de febrero del 2020). Sin embargo, podemos ir más profundo al afirmar que dicha admiración llevó a Duván a quedarse y vivir el proceso de formación, cuando agrega "yo estoy aquí es por usted" (Duván Restrepo. Entrevista. 4 de febrero del 2020). La primera frase nos habla de sentimientos fundantes del vínculo, la segunda relaciona el vínculo afectivo con el vínculo pedagógico, ya que habla de la volición, del porqué Duván se mantiene durante tantos años.

Por otro lado, ¿Sería posible que Duván realmente no admirara a Camero y tuviera un propósito oculto en su frase? Sería posible, pero también sería evidente, la puntada que nos da una presunción de veracidad en la afirmación, la admiración de Duván a Camero fue uno de los motores del proceso formativo o, dicho de otra forma, el vínculo afectivo propicia el vínculo pedagógico; es la amistad que conservan luego de más de 10 años, no solo entre ellos, sino con otros participantes. Esto, sumado a los vínculos afectivos que comienzan a expresarse en relación con "la profe", son señales que nos invitan a dirigir la mirada en esta dirección.

\section{Metodología}

Para esta investigación se llevó a cabo una selección basada en criterios simples, es decir, una muestra que el investigador seleccionó mediante juicio subjetivo, una serie de características que se definieron intencionadamente y a conveniencia de la investigación con rasgos comunes de la unidad 
de investigación (Goetz ,1998). La muestra de cuatro personas entrevistadas, de acuerdo con el problema formulado y la población referida, se enmarcó en los siguientes parámetros o criterios de selección:

1. Que haya participado del proyecto mínimo por 10 años de manera ininterrumpida.

2. Que actualmente tenga entre 15 y 25 años

3. Disponibilidad e interés en participar en el proceso investigativo. Además, se incluyeron a otros participantes de la comunidad pedagógica:

- Lina Tobón Londoño, fundadora y directora de la Corporación, que durante los 10 años en los que se enmarca la evaluación fue la profesora principal de los participantes.

- El padre o la madre de cada uno de los participantes.

Caracterización de las fuentes

A continuación, hago una breve presentación de las personas que participaron en la investigación,

\section{Sebastián Camero Galeano.}

Tiene 23 años, es estudiante de arquitectura de la Universidad Pontificia Bolivariana, beneficiario del programa "ser pilo paga" gracias al resultado de las pruebas ICFES. Ingreso al proyecto de Capoeira a los 10 años. En esta época era habitante del barrio Moravia, vivía en "el morro" por lo que en la época de reubicación tuvo que mudarse para el barrio Castilla, en donde habita actualmente. Fue educado por su mamá, ya que su padre murió cuando él era muy joven. Además, siempre ha vivido con su hermano, abuela, tío, tía y primo. Es uno de los participantes más antiguos del proyecto y pasó de ser estudiante para formar parte del equipo docente de la Corporación, ya que descubrió allí su vocación docente y amor por la música. En 2012 comienza a liderar uno de los proyectos más importantes de la Corporación, "La Batulata" a la cual hace referencia durante las entrevistas, como el lugar donde se sentía más a gusto y en donde pudo desarrollar la pasión por la música que hoy le acompaña. Actualmente participa en Baba Nkwa, proyecto de estudios musicales afrolatinos de la Corporación, ya no practica Capoeira. A lo largo del texto Sebastián será nombrado, Camero, la forma en que, por cariño, le dicen sus compañeros.

\section{Duván Emir Restrepo}

Tiene 23 años, comenzó a estudiar ingeniería de sistemas al salir del colegio, pero, actualmente, no estudia. Desea ingresar a la carrera de entrenamiento deportivo, educación física o alguna carrera afín. Ingreso al proyecto de Capoeira cuando tenía 10 años. Él y Camero eran compañeros del colegio El bosque, en el barrio Moravia. Creció en el barrio Moravia, en donde actualmente vive con su mamá, hermano, abuelo y otros familiares. Durante su infancia siempre vivió con su abuela y su abuelo, ya que sus padres vivían fuera de la ciudad, su abuela muere en 2018. Al igual que Camero, Duván pasa a integrar el equipo docente, ya que muestra gran vocación para el trabajo con niños y niñas. Comenzó a trabajar como monitor de las clases de los más pequeños, para luego asumir su propio proceso en el municipio de Belmira. Actualmente Duván continúa entrenando Capoeira y desea continuar con la carrera como capoeirista, sueña con llegar a ser Mestre. Dentro del mundo de la Capoeira es conocido como Papagaio (loro). A lo largo del texto se hace referencia a él como, Duván. 


\section{Emmanuel Herrera Londoño}

Emmanuel es llamado, dentro del mundo de la Capoeira, como, Bahiano; según él porque los habitantes de Salvador de Bahía son personas muy tranquilas y relajadas, y como él era muy tímido, decidieron nombrarlo así. Actualmente tiene 17 años, siendo el más joven de los tres informantes. Ingreso al proyecto de Capoeira a los 7 años. Actualmente se dedica a trabajar con su padre en un café de la familia, sin embargo, sueña con estudiar trabajo social. Al igual que Duván, asistió como monitor en la clase de los más pequeños durante varios años, encontrando allí su amor por el trabajo con comunidades. Sin embargo, decidió salir del proyecto antes de llegar a una graduación que le permitiera ser profesor. Actualmente está regresando a entrenar después de varios años sin hacerlo. Él y su papá fueron habitantes asiduos del barrio Moravia a pesar de que vivían en la ciudad de Bello. A lo largo del texto se hace referencia a él como, Emmanuel.

\section{Lina Marcela Tobón Londoño}

Tiene 35 años y practica Capoeira desde los 15 años, aproximadamente. Es cofundadora de la Corporación X y creadora del proyecto de Capoeira en el barrio Moravia. Su familia es originaria de Moravia, sin embargo, ella creció en el barrio Las Violetas y Buenos Aires. En el año 2007 realiza una especie de retorno simbólico a sus raíces, al acompañar la fundación del Centro de Desarrollo Cultural de Moravia. Actualmente es la directora de la Corporación X. Estudió un pregrado en educación física y es magíster en gestión cultural de la PUC de Minas Gerais, Brasil. Dentro de la Capoeira es conocida como Cafeína y tiene graduación de Instructora, el grado más alto dentro del grupo en Colombia, por lo que, además, lidera el grupo a nivel nacional. En el texto será nombrada Lina. Con Lina se realiza una entrevista que tuvo como objetivo vislumbrar el panorama en el que se ha desarrollado el proyecto y cuáles han sido las intenciones pedagógicas subyacentes. Dichas intenciones en ocasiones son conscientes, en otras habitan en la historia de la Corporación o en los principios filosóficos regentes. Además, se presenta como una oportunidad para evaluar las transformaciones a partir de su percepción de estas. Lina se concibe como fuente primaria, por ser la persona que no sólo ha acompañado más de cerca el proceso, sino que, es a partir de su intención pedagógica y personal, sus búsquedas e intereses que surge el vínculo pedagógico.

\section{Resultados}

A continuación, se presentan los hallazgos a través de cinco postulados: a) Primer postulado: el acto formativo implica que el aprendiz permita al Otro influir en la percepción que tiene de sí mismo; b) Segundo postulado: la norma, al integrarse, se percibe como voluntad de transformación; c) Tercer postulado: los referentes de progreso son motor del proceso de formación; d) Cuarto postulado: el Maestro o la Maestra es aquel que es reconocido por una comunidad como portador del arte. e) Quinto postulado: la participación de la familia es fundamental en el establecimiento de un vínculo pedagógico efectivo.

a) Primer postulado: el acto formativo implica que el aprendiz permita al Otro influir en la percepción que tiene de sí mismo

Se propone que, al hablar del encuentro en torno a la Capoeira se enmarque en el concepto de "formación" (Von Humbolt, citado por Sianes-Bautista, 2017 p. 101) ya que, como se expondrá, la Capoeira no sólo consiste en aprender un conocimiento o un gesto técnico, sino que implica la 
transformación integral del aprendiz. A la manera en que los alquimistas buscan transformar la piedra en oro, así mismo la Capoeira acompaña el proceso de transformación integral del ser. A diferencia del saber científico que observa y experimenta con "la cosa", la Capoeira por ser un saber íntimo que involucra cuerpo, mente y espíritu, exige al aprendiz que se involucre en una experiencia profunda y subjetiva con el saber, el aprendiz debe "realizar en sí" el saber. Así como expone Foucault (1982) en la hermenéutica del sujeto, al hablar sobre la espiritualidad el sujeto debe transformarse para acceder al saber. En relación con el concepto de "El Otro", Vergara (2012) cuando recupera a Bleichmar para decir que: "El Otro es definido como «el lugar desde el que se le aporta el código, es decir el lenguaje, las palabras que van a captar y a moldear por tanto sus necesidades... el Otro como el lugar del código". (p. 38).

Se encontró en las entrevistas que, aunque la autopercepción es un proceso íntimo que corresponde a la mirada que se tiene de sí mismo, esta reconoce y se relaciona de forma estrecha con la intención del Otro. Así, la autoridad, como una construcción ideal, a partir de las expectativas que "creo" que el mundo tiene sobre mí. Dicha idea se construye a partir de conversaciones, discusiones, gestos y otros elementos de la interacción cotidiana con aquel o aquello que considero como, autoridad. ¿Qué hace que perciba mi "timidez" o mi "falta de control" o mi "exceso de orgullo" como un problema de comportamiento que debe ser corregido?

Es entre comillas que se enuncia el calificativo del comportamiento, porque siempre está en relación con la opinión del Otro, una opinión creada como consenso. Es decir que, aunque lo que conseguimos percibir en las historias y opiniones de las personas entrevistadas, habita en el mundo de la autopercepción, estas nos están hablando de cómo la opinión de la autoridad ha influido en los demás. En palabras de Duván:

O sea, es que no es que, en el grupo, me hayan dicho como "Duván deje de ser tan hiperactivo, tan así"; no, o sea, yo creo que más que le digan a uno, más que le diga no ¿sí? Es más bien cómo saber entenderlo a uno, no entenderlo como que "¡Ay que pesar, ay dejémoslo así! No, sino que es que más bien como que hacerlo estrellar a uno con la realidad como que esos comportamientos no van en ese momento. (Duván. Entrevista. 4 de febrero del 2020).

Dicho consenso, a la luz de las observaciones realizadas, sucede a partir del encuentro entre pares y en el encuentro con la norma de comportamiento. El primer día que Duván llegó al aula, Camero le dice a Lina, “Seis horas en el colegio y ¿Acá, también? ¡No puede ser!” (Lina. Entrevista. 1 de marzo del 2020). Ante esta situación, Duván debió de tomar una decisión: o se transforma para ser aceptado bajo las normas de esta nueva colectividad a la que quiere pertenecer; o se va y busca un lugar en donde lo acepten como es. Se trata de una decisión que lo lleva a convertirse en aprendiz, una decisión que implica aceptar que debe embarcarse en un proceso de transformación personal. Es en este preciso momento, y en las repetidas ocasiones en que debe reafirmar dicha decisión, en las que el acto formativo sucede, la decisión que permite la entrada de la opinión externa en la percepción que tengo de mí mismo.

Esto permite plantear la siguiente cuestión: ¿Por qué Duván elige quedarse y transformarse? Sería atrevido querer dar una respuesta única a esto. Sin embargo, en las entrevistas se evidencia que Duván sentía amor y admiración por Camero. Es decir que aquel que llega a manifestar el límite es Camero, quien ya se había ganado el respeto de Duván y tenía permitido decirle cómo debía comportarse. Camero estaba investido por la autoridad que la admiración de Duván le daba. Lina cuenta que, en una ocasión, se encontraban discutiendo porque Camero se estresaba tanto con Duván que lo llegaba a maltratar, diciéndole "Cállese que la profesora está hablando, cualquier cosa de esas" 
- Entonces Duván le respondió: - "Camero, pero es que yo a usted lo admiro mucho hermano, es que yo estoy aquí es por usted". (Lina. Entrevista.1 de marzo del 2020).

Camero cumple un doble papel en la vida de Duván, como fuente de inspiración y como límite que le ayuda a controlar aquello que le genera displacer y dificultades a nivel relacional. Frente a la presencia de emociones negativas y rechazo del grupo en relación con la forma de ser de Duván, él manifiesta: «Sí, pues, en el momento no me acuerdo quién, porque ha pasado mucha gente por el grupo, pero era mucho de que, "No quiero entrenar con Papagaio. No quiero hacer los ejercicios con Papagaio". Entonces, la gran mayoría de los ejercicios me tocaba hacerlas con Duende, con Cafeína, con los profes". (Duván. Entrevista. 4 de febrero del 2020).

Uno y otro, autoridad y referencia, se apoyan y fortalecen entre sí al aconsejar, amar, apoyar y confrontar. Como se mostrará, la confrontación juega un papel vital, en el proceso de formación integral de los participantes. Pues, es en la confrontación que se plantean y corroboran los límites, las reglas del juego. Se ha mencionado hasta el momento una forma de la autoridad, en la que es el par quien se convierte en autoridad. También es importante señalar la importancia que tiene la relación con los códigos y normas de la Capoeira, códigos que demandan un respeto al momento, reconocer que todo tiene su espacio. Un orden en la palabra, que indica que se escuche y se hable cuando es debido. Un orden situacional, en relación con el espacio, consciencia del cuerpo en relación con los elementos del espacio. Como dice Lina: "Él [Duván] iba a hacer un ejercicio y literal todos nos alejábamos, nos pegábamos al espejo y él salía. Yo no sé cómo lograba que un movimiento en el que tenía mucho espacio, siempre le pegaba a alguien. O sea, era torpe con el cuerpo». (Lina. Entrevista. 1 de marzo del 2020) En este caso la autoridad no es otro ser humano, sino que está dada por pautas de conducta establecidas de manera colectiva y consensuada.

Estas normas son transmitidas por "la profe", quien las conoce, y es reconocida como la portadora, más no la creadora de estas. Esto es importante, ya que una afrenta a las normas no es una afrenta a la autoridad, es un desconocimiento del valor que tiene el consenso y la ancestralidad presente en dichas normas, que se integran a un punto en el que cuestionarlas es inconcebible. Además, las normas tienen una utilidad visible dentro del juego de Capoeira. Es fácil reconocer que romperlas, es acabar con la posibilidad de que se desarrolle el juego. El código que se establece para saber quién puede jugar y quién no. Por ejemplo, dice que debes dirigirte a un lugar específico: dos jugadores arrodillados a los pies de los instrumentos musicales (la batería), se miran a los ojos y esperan que el Berimbau Gunga, quien dirige la roda les haga la señal de que ya pueden jugar. Romper esta regla es impedir que el juego como tal se desarrolle, sería como desconocer los limitados movimientos en las fichas del ajedrez o tomar el balón con la mano en el futbol. Quien ama jugar, respeta las reglas. 


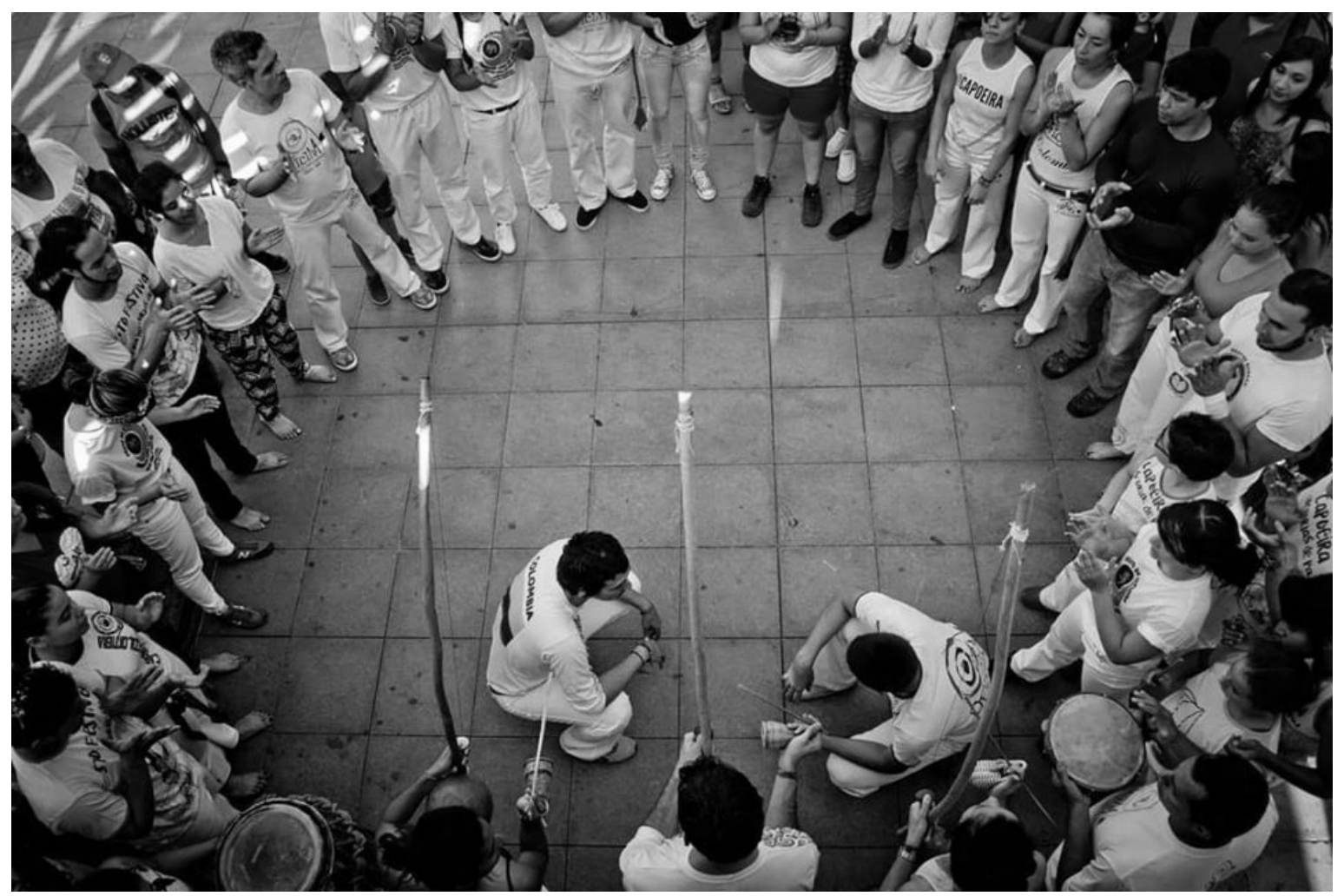

Imagen 1. Duván y Duende (el profe) arrodillados al pie del berimbau, esperando permiso de Lina, en el Berimbau Gunga, para jugar. Tomada del registro fotográfico de la Corporación X.

\section{b) Segundo postulado: la norma, al integrarse, se percibe como voluntad de transformación}

Polaino-Lorente (1988) hace una distinción entre voluntad y deseo, “...seguir los deseos no implica ejercicio alguno de voluntad, significa un dejarse arrastrar o ser arrastrado" (P. 73). Dicho conflicto entre voluntad y deseo se hace evidente en la historia de Emmanuel. Su deseo era permanecer en casa, motivado por el miedo a relacionarse, que lo llevaba incluso a enfermarse y somatizar el miedo. Es la voluntad de su papá la que lo lleva a participar de las primeras clases de Capoeira. Sin embargo, a él le gusta tanto lo que allí sucede, se enamora de la Capoeira y del grupo a un nivel en el que la norma, socializar, se le vuelve un propósito, un motor de transformación.

En el momento en que la voluntad de transformación emerge, la persona reconoce la falta desde una opinión propia y no solo como crítica externa. El reconocimiento de la falta se convierte en potencia, ya que en el afán por dar solución a aquello que genera displacer, se activa un impulso vital, la voluntad, que es fundamental en el proceso de formación. La mirada está puesta en aquello que la práctica o la comunidad a la que deseamos pertenecer demanda, pero en sentido de demanda propia. Ahora no es alguien más que le pide a Duván que tome conciencia de su cuerpo en el espacio, o a Emmanuel que interactúe con otros, o a Camero que respete a sus mayores, ahora pasa a ser parte de sus propias búsquedas personales.

Se le plantea a Emmanuel la pregunta de si le parece importante haber dejado de ser tímido, a lo que responde: "Sí, total, porque yo no hablaba nada ni siquiera en clase, yo tenía mis amiguitos cercanos y ya, no hablaba con nadie más y si es muy importante no hacerlo porque cuando uno interactúa, uno conoce. Entonces es muy, muy, importante" (Emmanuel. Entrevista. 27 de marzo de 
2020). La satisfacción por haberse transformado no solo nace de una necesidad por complacer al otro, sino que ahora son sus propias metas las que se están cumpliendo:

Mi papá estaba en esas clases conmigo, la idea era como que yo fuera perdiendo la timidez, y llegó un momento en que él se salió y fue muy, muy teso, pero las clases cambiaron a los martes y jueves y los sábados y yo ya seguí mucho tiempo y la timidez se fue yendo, pues, fui cogiendo la confianza (Emmanuel. Entrevista. 27 de marzo de 2020).

c) Tercer postulado: los referentes de progreso son motor del proceso de formación.

Cuando la comunidad educativa presenta referentes de progreso al alumno, pero más importante aún, cuando estos referentes comienzan a habitar en los sueños y las fantasías de los aprendices, es cuando el cansancio del entrenamiento, las dificultades del camino o las críticas de otros dejan de importar, y la voluntad se fortalece y se vuelve un propósito profundo por llegar, por alcanzar. Los referentes son presentados entonces como personas que han logrado lo que el aprendiz quisiera lograr. En los tres casos que presentamos, los referentes estaban en constante tránsito con los invitados internacionales que año tras año venían a la ciudad y con los compañeros que llevaban más tiempo en el proceso.

Cuando Duván sabe por primera vez de la existencia del grupo, con lo primero que se encuentra es con un brasileño, Neguinho, quien, en palabras de Duván "De un bíceps tenía a Camero colgado y en el otro tenía a Cafeína" (Duván. Entrevista. 4 de febrero del 2020). No solo se sienten inspirados por los viajeros que llegan contando historias, cantando, jugando bien y volando por los aires en sus acrobacias, sino que además los compañeros comienzan a viajar y a traer historias de otros lugares. "Yo me acuerdo de que, el primero que viajó de los amigos fue Maca y cuando Maca viajó pues todos como, "marica hay que viajar"" (Emmanuel. Entrevista. 27 de marzo del 2020). Los sueños de los alumnos entonces comienzan a llenarse de otras tierras, de otras lenguas, de otros mundos posibles.

El círculo social de los participantes crece y empiezan a tomar nuevos referentes "Ya en el momento que entro pues ya empiezo a relacionarme con niños de mi edad, adolescentes, adultos ¿Sí? Ya después con el tiempo empiezan a llegar eses experiencias de conocer a otros profesores de la ciudad, profesor Curioso, profesor Pescador" (Duván. Entrevista. 4 de febrero del 2020). No siempre quien se supone debería ser la autoridad ejerce como tal. Como se mostró en la relación de Duván y Camero, requiere del vínculo amoroso para que esto suceda, y con estos nuevos viajeros, como Neguinho, el vínculo se da casi de manera automática por la admiración que producen en ellos. La importancia del vínculo nos da pie para hablar sobre la ética docente y las relaciones de poder que se establecen en el acto formativo, sin embargo, esta elaboración ética excede los objetivos de esta investigación.

Hasta aquí se pueden observar tres mecanismos que participaron en el proceso de transformación: la relación con la autoridad, la integración de la norma y la inspiración nacida del encuentro con referentes. Los postulados cuatro y cinco, son una suerte de caracterización de la comunidad en la que el participante vive su proceso de transformación. El cuarto postulado se enfoca en el maestro o la maestra y el quinto habla sobre la importancia de la familia en dicho proceso de transformación.

d) Cuarto postulado: el Maestro o la Maestra es aquel que es reconocido por una comunidad como portador del arte.

Ser Maestro o Maestra implica un reconocimiento que le otorga el título de portador del «buen gusto» en sentido Kantiano, tal como lo recupera Villa (2017) del pensamiento de Gadamer: "Bajo el signo del buen gusto se da la capacidad de distanciarse respecto a uno mismo y a sus preferencias privadas. Por su esencia más propia el gusto no es pues cosa privada sino un fenómeno social de 
primer rango" (pg. 86). Es decir, conserva y cuida del saber construido por la comunidad a través de la historia, convirtiéndose en autoridad que puede decir cómo se hace y cómo no se hace. Así, Maestro o Maestra no es cualquier persona que enseña, es un título que habla del estado más alto de conocimiento en la Capoeira. Se extrae de la Capoeira en esta tesis, como un intento por integrar el valor que tiene, a nuestra idea de Maestro y Maestra en el acto formativo de forma general.

Por su parte, si bien el reconocimiento puede jugar en el ámbito de la vanidad, y servir de alimento a fines comerciales, es, además, un ritual de paso en el que los aprendices simbolizan el valor del camino recorrido. Lo que entra en juego aquí son los valores que el grupo porta, cada comunidad de capoeiristas procura la formación de diferentes principios en sus aprendices y es a partir de dichos principios que se reconoce la transformación. Dado que el acto formativo carece de moral y puede ser arma útil para fines moralmente opuestos. Se hace necesario de nuevo, plantear en futuras investigaciones, una pregunta por la ética de la enseñanza.

Por otro lado, si bien en la Capoeira contemporánea el nivel de Mestre es una graduación que otorga otro Mestre, dicha graduación debe de ser reconocida por una comunidad. Es allí en donde los debates éticos propician el consenso, que llega para equilibrar las posibles arbitrariedades. En múltiples ocasiones esto no sucede, no hay consenso por lo que la graduación pasa a ser un acto ficticio que no repercute en la comunidad capoeirista. En algunas ocasiones, en cambio, una persona sin haber recibido su cuerda ya es llamada por los demás capoeiristas como, Mestre.

En consecuencia, la manifestación artística aquí no se limita a la experiencia estética, sino que adquiere valor al convertirse en alegoría de un saber ancestral, del sentir e historias de un pueblo. El maestro es el heredero y guardián de un saber cultivado por generaciones y cuidado con celo. Cuando la rueda de Capoeira sucede, se pone en escena la historia de un pueblo, los cantos narran el dolor y las alegrías; y los ritos que allí se desenvuelven evocan los sentires que habitan en la base misma de la cultura popular brasileña.

El maestro en la Capoeira que llega a Moravia es un ser que se entrega al cuidado de la tradición y al servicio de la gente. Por esto es reconocido por la comunidad. Dicho espíritu de entrega la podemos hallar en la apuesta pedagógica expuesta en el segundo postulado, que, si bien es un legado que, heredado de las tradiciones brasileñas de matriz africana, en las que los mayores y los ancestros son la columna vertebral de las comunidades. Dicha creencia, además, habita en los principios de Lina y de la Corporación. Su objeto, su propuesta de valor y su espíritu es el servicio, el arte que porta el Mestre.

e) Quinto postulado: la participación de la familia es fundamental en el establecimiento de un vínculo pedagógico efectivo

El vínculo arroja luces que invitan a ver su gran importancia. Desde la primera entrevista con Duván, cuando en un lapsus del lenguaje, llama a Lina mamá: "Era la primera vez que yo salía solo, o sea, solo en el sentido de que yo siempre salía con mi abuela, con mi abuelo ¿Sí? No, en estos momentos salgo solo, solamente con mi mamá, digo, con Cafeína. Bueno, prácticamente es la mamá de la generación en la que yo crecí". (Duván. Entrevista. 4 de febrero del 2020). Es importante señalar que los tres casos analizados, corresponden a familias monoparentales. En el caso de Camero y de Emmanuel, la presencia de la mamá y el papá, respectivamente, es grande, se trata de padres presentes y comprometidos con el proceso de sus hijos; al igual que en el caso de Duván, con quien sus abuelos cumplen con la labor de padres comprometidos. Este compromiso y presencia de sus padres es una afirmación que hace Lina: "Ustedes también han sido muy acompañados por sus familias» (Lina. Entrevista. 16 de marzo). 
Sería muy atrevido afirmar que los participantes fueron movidos por ausencias en su hogar, a pesar de las particularidades que presentan, pues, ellos así no lo manifiestan. Mas, se hace evidente, que las familias encuentran en Lina una aliada para la formación de sus hijos, la invitan de forma clara a que participe de la intimidad de sus hogares, invitándola a eventos de celebración y de duelo. Pero, sobre todo, presentándole sus propias limitaciones, confiando en que en ella podrían encontrar una aliada que supliera estas. Como dice Lina: "Tengo incluso el día, la hora, la luz, cuando me encontré a la mamá de Camero saliendo del centro cultural y ella me dice: "Café, estoy muy triste, estoy muy triste, ese muchacho lleva no sé cuántos años perdidos, no quiere hacer nada por la vida"»» (Lina. Entrevista. 1 de marzo del 2020).

Camero era evidentemente un niño a quien su mamá cuidaba con esmero, su presentación personal era buena, el trato con el otro, la educación. Como dice Lina: "A mí me sorprendió mucho cuando conocí la casa de Camero, porque Camero cuando llegaba a clase era siempre organizadito, olía rico, él tiene pinta de ser un chico de buena familia, de buen trato. Pero cuando llegas a la casa de él, no tenía ni siquiera cama, enrollaba un colchón que abría en un espaciecito [Sic] como de dos baldosas». (Lina. Entrevista. 1 de marzo del 2020). Pese a estas condiciones de carencia, la atención de la familia estaba presente en Camero, como en los tres casos que se han presentado.

\section{Discusión y conclusiones}

La participación de la Corporación X en el proceso de transformación de los participantes es evidente, gracias a un proyecto de pedagogía comunitaria que ha venido madurando, apoyado por el dialogo constante entre los diferentes actores de la comunidad educativa. Gestando procesos que se dirigen a la formación integral y no solo a la transmisión de un saber técnico. Los aportes de la Corporación X, entonces, ofrecen información valiosa para la construcción de nuevas propuestas, que partan de una comprensión de la formación como un encuentro entre los seres, que escucha las voces de aquellos que desean transformase desde la voluntad y la pasión, concibiendo a los y las participantes como potencia y no como carencia.

La Corporación X, se entrega a transformarse junto con la comunidad, se concibe a sí misma como una fuerza más de dicha comunidad y no como un agente externo, que llega a ejecutar programas asistenciales. Es así que, cuando hablamos de las transformaciones que vivió el barrio Moravia en la última década, de inmediato emergen rostros de distintas latitudes, muchas voluntades que dirigieron la mirada al barrio y fueron rápidamente haciendo de él lo que es hoy. No solo fueron los habitantes del barrio o la gente de Medellín, Moravia ha sido el lugar de acogida de personas del mundo entero que llegaron para dar su granito de arena en la transformación del barrio.

Es por esto por lo que, aunque la participación de la Corporación X en la transformación del barrio es evidente en los discursos de sus habitantes, en especial de los líderes y lideresas, difícilmente podríamos señalar los mecanismos o los cambios concretos sin un estudio dirigido a la población en general, y que excede los alcances de la presente investigación. La Corporación X se suma con su voluntad, sus sueños y sus apuestas a una situación y una coyuntura particular. Sería absurdo querer acreditar loas personales a aquello que fue importante gracias al pensamiento comunitario, interdisciplinario y multidimensional. 


\section{Referencias}

Fernández, S. (2017) Evaluación y aprendizaje. España

Foucault, M. (1982) La hermenéutica del sujeto. Francia

Goetz, J.P. y LeCompte, M.D. (1998) Etnografia y diseño cualitativo en investigación educativa. Evaluación del diseño etnográfico. Morata.

Polaino-Lorente, A. (1988). Dimensiones motivacionales y cognoscitivas de la educación de la voluntad. Dossat,

Ramírez, J.P. (2019) Moravia trazó su ruta para la historia y la cultura. Revista del Colombiano. https://www.elcolombiano.com/antioquia/medellin-antes-y-ahora-historia-del-barrio-moraviaHJ11180315

Sianes-Bautista, A. (2017) Bildung: concepto, evolución e influjo en la pedagogía occidental desde una perspectiva histórica y actual. Revista Española de Educación Comparada. (30), 99-111.

Vergara, C. (2012) El niño hoy: una perspectiva clínica y pedagógica. En clave social. Enero - Junio de 2012. 1(1), 58-66. http://repository.lasallista.edu.co:8080/ojs/index.php/ENClave/article/view/184/92

Villa, J. A. (2017) El gusto como discernimiento en Gadamer. Signos filosóficos, 19(37), 66-89. 